\title{
Can immature granulocyte count and hemogram indices be good predictors of urinary tract infection in children?
}

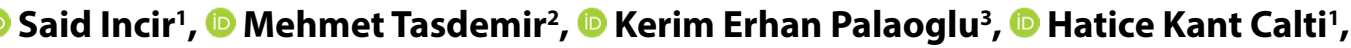 \\ (D) Arzu Baygul ${ }^{4}$, (1) Ilmay Bilge ${ }^{2}$
}

'Department of Clinical Laboratory, Koc University Hospital, Istanbul, Turkey

${ }^{2}$ Division of Pediatric Nephrology, Department of Pediatrics, Koc University Faculty of Medicine, Istanbul, Turkey

${ }^{3}$ Department of Clinical Laboratory, American Hospital, Istanbul, Turkey

${ }^{4}$ Department of Bioistatistics, Koc University Faculty of Medicine, Istanbul, Turkey

\begin{abstract}
Objectives: A left shift in a complete blood count (CBC) indicates the presence of immature granulocytes (IGs), and an elevated count is associated with a bacterial infection. The aim of this study was to evaluate the role of the IG count and inflammatory CBC parameters and indices in children with a urinary tract infection (UTI).

Methods: In this retrospective study, 55 children with a UTI (patient group) and 47 children with no infection matched for age and sex (control group) were enrolled. The symptoms, clinical findings, CBC parameters, and platelet-to-lymphocyte ratio (PLR), neutrophil-to-lymphocyte ratio (NLR), systemic immune-inflammatory index (SII), and urinalysis findings were recorded. The C-reactive protein (CRP) level, urine culture results, and radiological data for the UTI group were also noted and analyzed.

Results: The IG count; leukocyte, platelet, and neutrophil counts; and the CRP value were higher in the patient group than in the control group $(\mathrm{p}=0.001,0.001,0.035,0.001$, and $<0.001$, respectively). The NLR, PLR, SII, and mean platelet volume were not significantly different between the groups. The median hemoglobin level was significantly lower in the patient group than in the control group $(10.9 \mathrm{~g} / \mathrm{dL}$ vs. $12.2 \mathrm{~g} / \mathrm{dL} ; \mathrm{p}=0.004)$. The area under the curve values for CRP and counts of IG, leukocytes, and neutrophils were 0.851 (95\% confidence interval [CI]: $0.761-0.917), 0.736$ (95\% Cl: $0.633-0.823), 0.714$ (95\% Cl: 0.609-0.804), and 0.670 (95\% Cl: 0.564-0.765), respectively.

Conclusion: The IG count is an easily obtained measurement that can be utilized alongside the CRP value and other indicators to create better predictive value in the management of UTI.
\end{abstract}

Keywords: Acute-phase reaction, blood cell count, child, granulocytes, urinary tract infection

A urinary tract infection (UTI) is one of the most common bacterial infections to occur in children. Prompt diagnosis and efficient antimicrobial interventions are required to limit renal scarring and progressive kidney injury [1]. Urine dipsticks and microscopic analysis continue to be valuable tools for a presumptive diagnosis of UTI. Microscopic urinalysis is also used to ascertain the number of leukocytes in the urine, which is a sensitive indicator of inflammation associated with infection $[1,2]$. As a consequence of the suboptimal sensitivity and specificity of urinalysis parameters, alone and in combination, a urine culture is considered the most efficient test for the diagnosis of UTI [2]. Significant bacteriuria in a patient with a negative urinalysis may reflect a false-positive urine culture due to asymptomatic bacteriuria or contamination [3]. Due to this potential for confusion in the diagnosis of UTI, it is vital to support the diagnosis with clinical (symptoms and examination findings), laboratory (acute-phase reactants), and radiological findings (ultrasonography or dimercaptosuccinic acid [DMSA]).

Address for correspondence: Said Incir, MD. Department of Clinical Laboratory, Koc University Hospital, Istanbul, Turkey Phone: +90 5322183766 E-mail: sincir@kuh.ku.edu.tr ORCID: 0000-0002-7700-7388

Submitted Date: February 22, 2021 Accepted Date: April 20, 2021 Available Online Date: September 10, 2021

${ }^{\circ}$ Copyright 2021 by International Journal of Medical Biochemistry - Available online at www.internationalbiochemistry.com OPEN ACCESS This work is licensed under a Creative Commons Attribution-NonCommercial 4.0 International License. 
The acute phase response of the body to an infectious agent generally manifests as fever, leukocytosis, and an increase in certain plasma proteins. Previous studies have provided potential non-invasive biomarkers that may be used to identify a systemic inflammatory response, including $\mathrm{C}$-reactive protein (CRP), procalcitonin, erythrocyte sedimentation rate (ESR), cytokines, chemokines, cell surface antigens, and immunoglobulins and antibodies for natural killer cells [4].

As well as acute-phase reactants, certain hemogram parameters (neutrophil count, lymphocyte count, neutrophil percentage, lymphocyte percentage, red blood cell distribution width [RDW], platelet count, platelet hematocrit [PCT], platelet distribution width [PDW] and platelet-large cell ratio [P-LCR]) can also be early diagnostic markers of inflammation and bacterial infection [5]. In addition, certain complete blood count (CBC) ratios, such as the platelet-to-lymphocyte ratio (PLR), neutrophil-to-lymphocyte ratio (NLR), and the systemic immune-inflammatory index (SII) values can also be indicators of inflammation [6].

Automated hematology analyzers can detect acute inflammation by providing detailed data of a total white blood cell (WBC) count, absolute neutrophil count (ANC), and band formation, which may also provide early signs of inflammation or infection. A left shift in the $C B C$ reflects the presence of immature granulocytes (IGs), which mirror the neutrophil band, and an elevated count is associated with a bacterial infection. The IG count, which can be calculated by counting the cells in the area above the neutrophil granulocytes on a $3 \mathrm{D}$ graph, is the combination of promyelocyte, myelocyte and metamyelocyte cells that mature with myeloid cells, and may be useful to a prompt diagnosis of bacterial infection [7-9]. Although a CBC is a fast, cost-effective, and reliable test, very few validation studies have been published on its value to UTI diagnosis. It could prove to be very useful.

The present study was designed to investigate the role of the IG count and other inflammatory CBC parameters and indices in the presence of systemic inflammation/infection in a patient diagnosed with a UTI. The area under curve (AUC) values of $C B C$ parameters and indices were calculated to evaluate the prognostic value.

\section{Materials and Methods}

\section{Study design}

A total of 55 children diagnosed with a UTI (patient group) and 47 healthy children with a homogeneous distribution in terms of age and gender (healthy control group) who were admitted to the pediatric outpatient clinic of a single hospital between May 2018 and May 2019 were included in this retrospective study. The symptoms, clinical findings, and the results of CBC and urinalysis for the whole study population, as well as CRP level, urine culture, and radiological data for the UTI group, were analyzed.

All of the data were assessed by 2 physicians (Si and MT) as well as a third researcher (HKÇ) to detect any difference in in- terpretation. The exclusion criteria for the research were age $>18$ years, recent use of any medication, or the presence of a bacterial infection other than a UTI, a history of hemolysis or coagulopathy in the patient's sample data, a diagnosis of a chronic disease (such as autoimmune disease, obesity, diabetes, chronic renal failure), and the presence of a hematological or oncological disease.

The diagnosis of a UTI was made according to guidelines based on symptoms (including fever, nausea, vomiting, dysuria, and abdominal pain), clinical findings on examination (including costovertebral angle or abdominal tenderness), the presence of pyuria (more than 10 leukocytes per high power field, nitrite and leukocyte esterase positivity), and a positive urine culture. Radiological assessment records (ultrasonography and voiding cystourethrography) and DMSA scintigraphy were also evaluated for any possible underlying condition or damage. Children who were examined in a routine check-up at the outpatient clinic with no infectious condition and with a normal urinalysis were accepted into the healthy control group.

The laboratory data analyzed were obtained on the first sampling after admission and before medication. The IG count, IG percentage (IG count divided by $\mathrm{WBC} \times 100$ ) and other hemogram parameters were assessed using a Sysmex XN3100 device (Sysmex Corp., Kobe, Japan). The CRP level was determined using a Roche Cobas-6000 analyzer (F. HoffmannLa Roche Ltd., Basel, Switzerland).

The between-group difference was assessed for the variables of age, sex, CRP level, leukocyte count, IG percentage and count, neutrophil count, lymphocyte count, RDW, platelet count, PCT, PDW, P-LCR, PLR, NLR, SII (neutrophil*atatelet/ lymphocyte), and leukocyturia and erythrocyturia assessments.

The protocol for the present study was reviewed and approved by the ethics committee of Koç University (approval number: 2020.151.IRB2.041). The committee exempted the authors from the need to obtain any additional informed consent due to the retrospective design of the research.

\section{Statistical analysis}

The statistical analysis was performed using MedCalc Statistical Software version 12.7.7 (MedCalc Software bvba, Ostend, Belgium; http://www.medcalc.org; 2013). The Shapiro-Wilk test was applied to examine the normality of the distribution of continuous variables. Descriptive statistics were presented using the mean and SD for normally distributed variables and the median (minimum-maximum) for non-normally distributed variables. Non-parametric statistical methods were used for values with skewed distribution. The Mann-Whitney $U$ test was used to compare 2 non-normally distributed groups, and normally distributed groups were evaluated using the Student t-test. A chi-squared test was used to assess categorical variables and the results were expressed as observation counts (and percentages). Associations between the IG count, 
and other parameters were assessed using Spearman correlation analysis. Variables with a $p$ value of $<0.05$ in the univariate analysis were tested using multivariate linear regression analysis to identify the important independent factors influencing IG count.

Receiver operating characteristic analysis was performed to evaluate the predictive ability of the parameters. Statistical significance was defined as a 2 -sided $p$-value of $<0.05$.

\section{Results}

The demographic, clinical, and radiologic characteristics of the patients and controls are presented in Table 1. There was no significant difference in age, gender, weight, or height. The median age was 2.61 years (range: $0.05-14.9$ years) and 5.01 years (range: $0.05-15.9$ years) in the patient and control groups, respectively. As presented in Table 1, the most frequent presenting symptoms in the patients were dysuria, vomiting (each 16.4\%), and abdominal pain/restlessness (12.7\%). On examination, $40 \%$ of the patients had a fever and $7.3 \%$ had suprapubic tenderness. Urinary system ultrasonography was performed on $20 \%$ of patients and showed normal findings $(5.5 \%)$, bladder disease $(5.5 \%)$, ureterocele $(3.6 \%)$, a duplicated collecting system (3.6\%), small kidney and hydronephrosis (both $1.8 \%$ ). Of 55 patients, voiding cystourethrography was performed in 14 (25.4\%), which revealed vesicoureteral reflux in 8 patients $(14.5 \%)$, and 5 underwent DMSA scintigraphy (9.0\%), which demonstrated unilateral hypoplasia in $2(3.6 \%)$ and renal scarring in $1(1.8 \%)$ patient.

The median IG count was significantly higher in the patients than in the controls ( 0.04 vs. $0.02 ; p=0.001)$, but there was no significant between-group difference in the IG ratio $(p=0.053)$ (Table 2). The NLR, PLR, SSI, and MPV values were also not significantly different. The median leukocyte, platelet and neutrophil counts, as well as the CRP value, were higher in the patient group than in the control group $(p=0.001,0.035,0.001$, and $<0.001$, respectively). The median hemoglobin level was significantly lower in the patients $(10.9 \mathrm{~g} / \mathrm{dL}$ vs. $12.2 \mathrm{~g} / \mathrm{dL}$; $\mathrm{p}=0.004)$. The leukocyte esterase result was $1+$ in $5(9.1 \%), 2+$ in 10 (18.2), and $3+$ in 37 (67.3\%) patients, and the nitrite find-

Table 1. Demographic, clinical, and radiological features of the patients and the controls

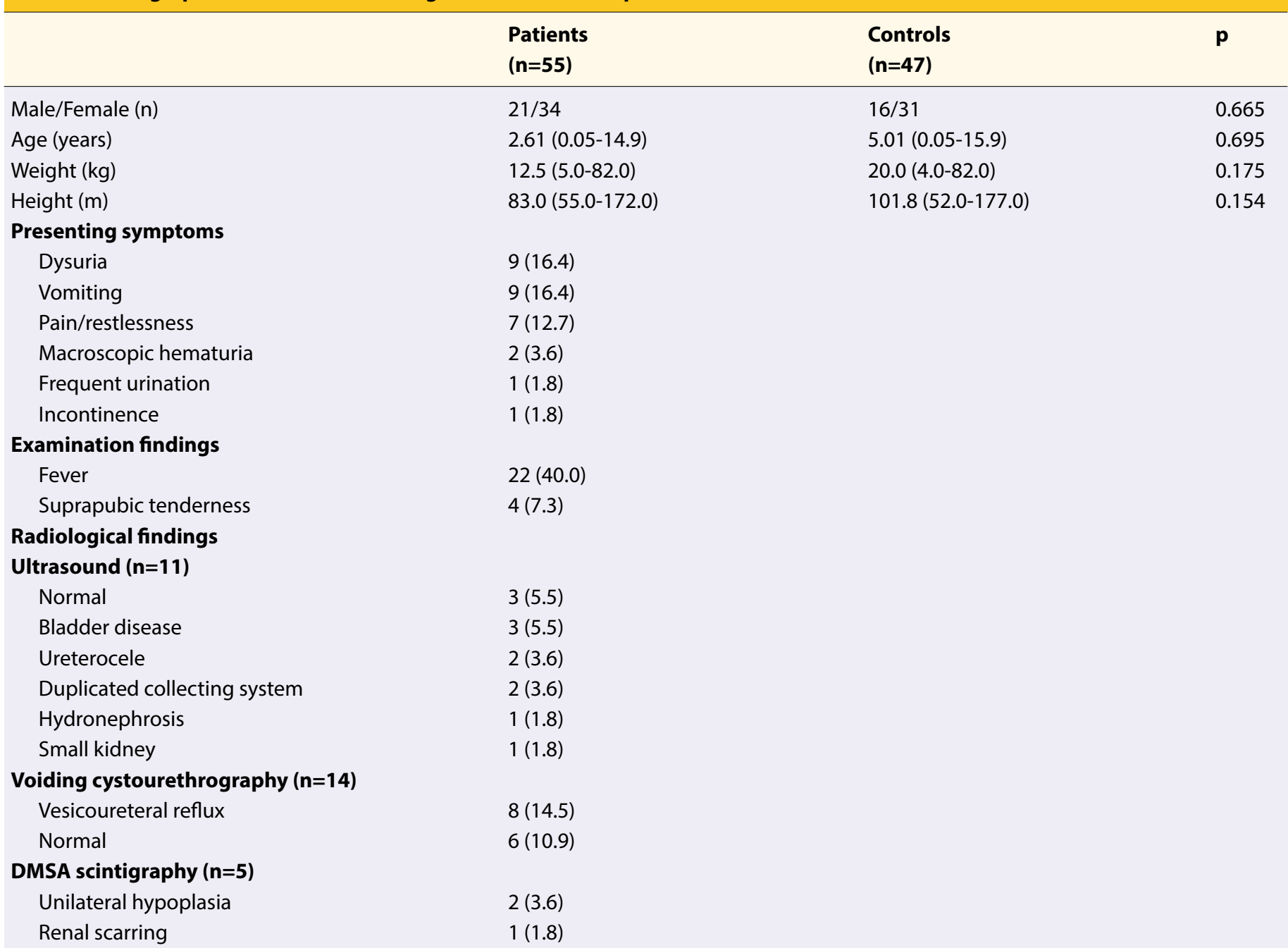


Table 2. Inflammatory parameters of the patients and the controls

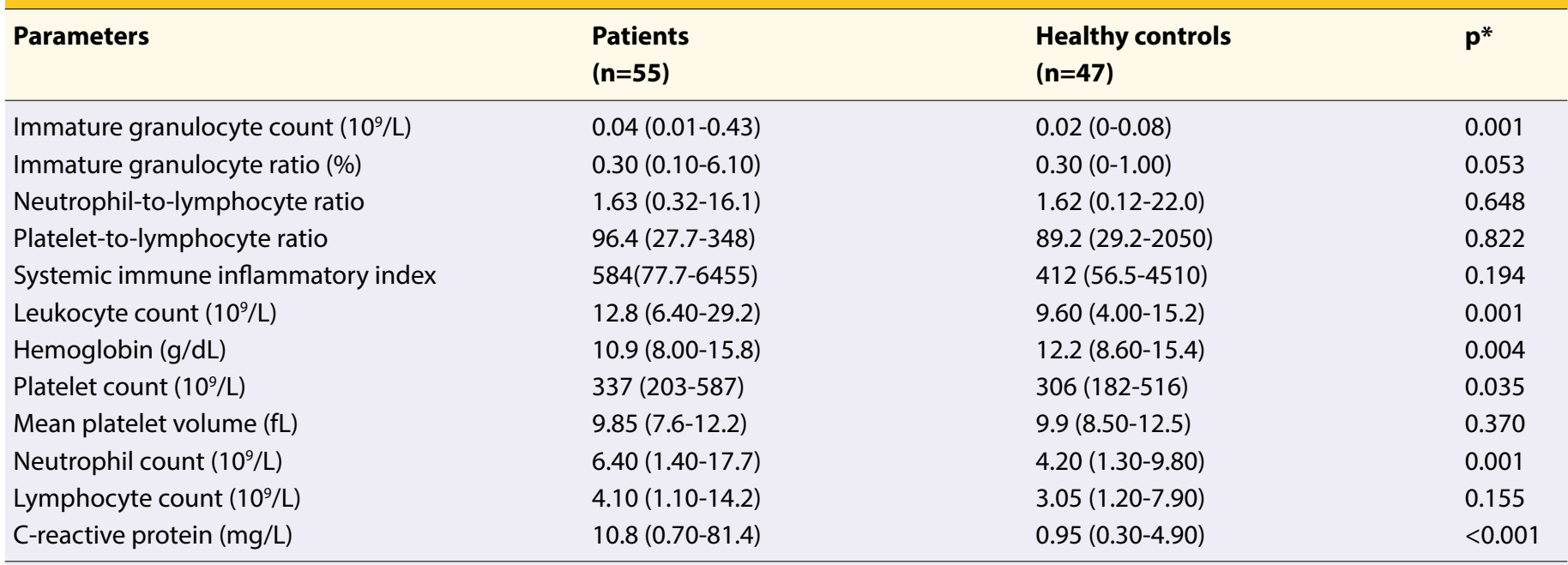

Data are presented as the median (minimum-maximum). $\mathrm{p}^{*}$ : Comparison between the patients and healthy controls using the Mann-Whitney U test.

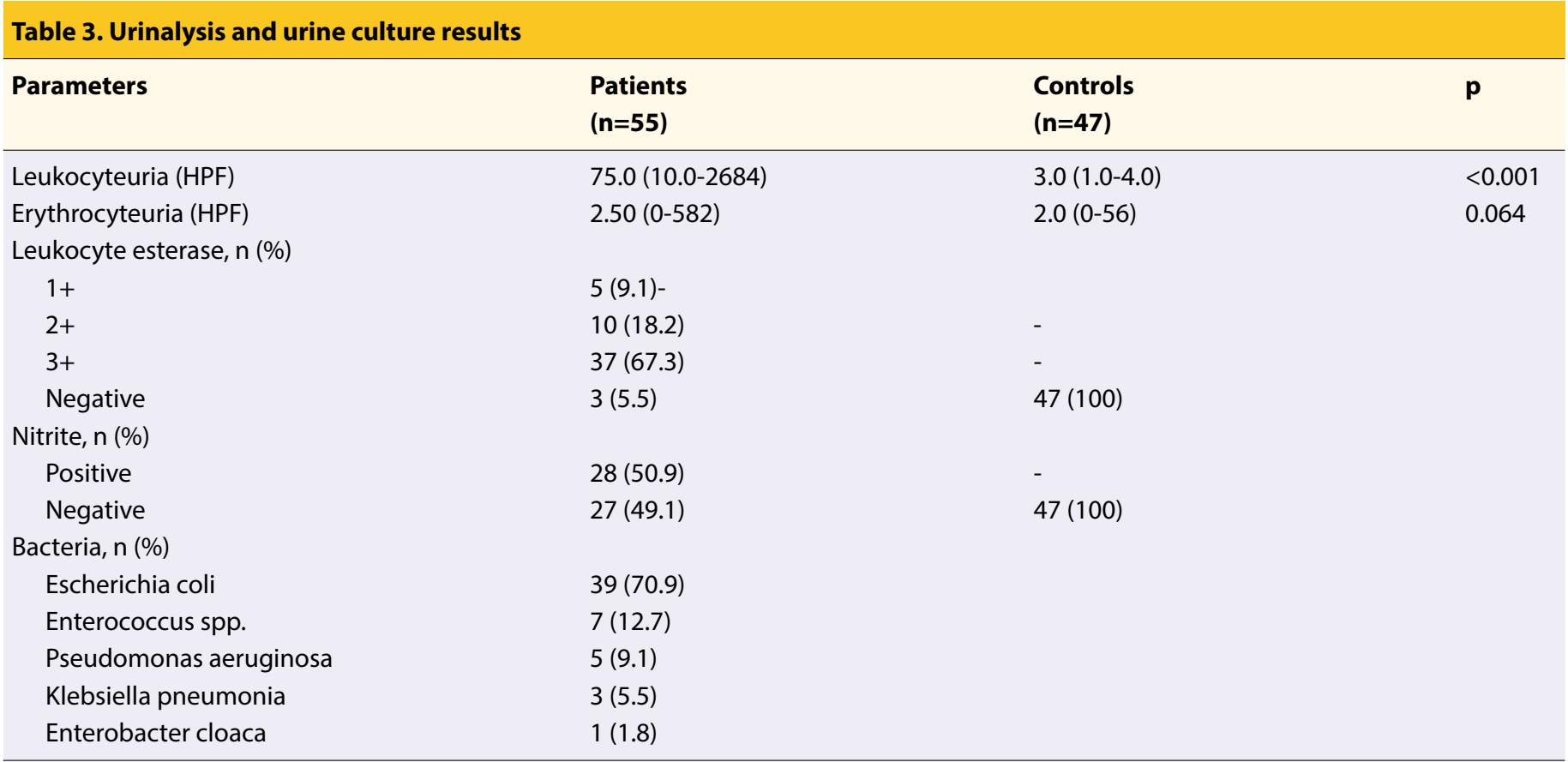

Data are presented as the median (minimum-maximum) or $n$ (\%). HPF: High power field.

ing was positive in 28 (50.9\%) patients. The most common microorganisms found were Escherichia coli, Enterococcus spp., and Pseudomonas aeruginosa, as shown in Table 3.

Positive correlations between the IG count and inflammatory parameters were observed in the SII, NLR, and CRP values. The CRP level was an independent predictor $(\beta=0.338 ; p=0.025)$ for the IG count (Table 4).

The AUC values for the CRP level, IG count, and leukocyte and neutrophil counts were 0.851 (95\% Cl:0.761-0.917), 0.736 (95\% Cl: $0.633-0.823), 0.714$ (95\% Cl: 0.609-0.804), and 0.670 (95\% Cl: $0.564-0.765)$, respectively. The cut-off value of the IG count as a predictor of UTI was $>0.03$, with a sensitivity and specificity value of $65.45 \%$ (95\% Cl: $51.4-77.8)$ and $65.96 \%$ (95\% Cl: $50.7-$ 79.1), respectively (Fig. 1).

\section{Discussion}

The results of the present study demonstrated a significant increase in IG, leukocyte, neutrophil, and platelet counts, as well as CRP level and a significant decrease in hemoglobin levels in children with a UTI. Additionally, a notable correlation between the IG count and the SII, NLR, platelet count, and CRP level was observed. 
Table 4. Correlations between the immature granulocyte count, inflammatory parameters, and hemoglobin level

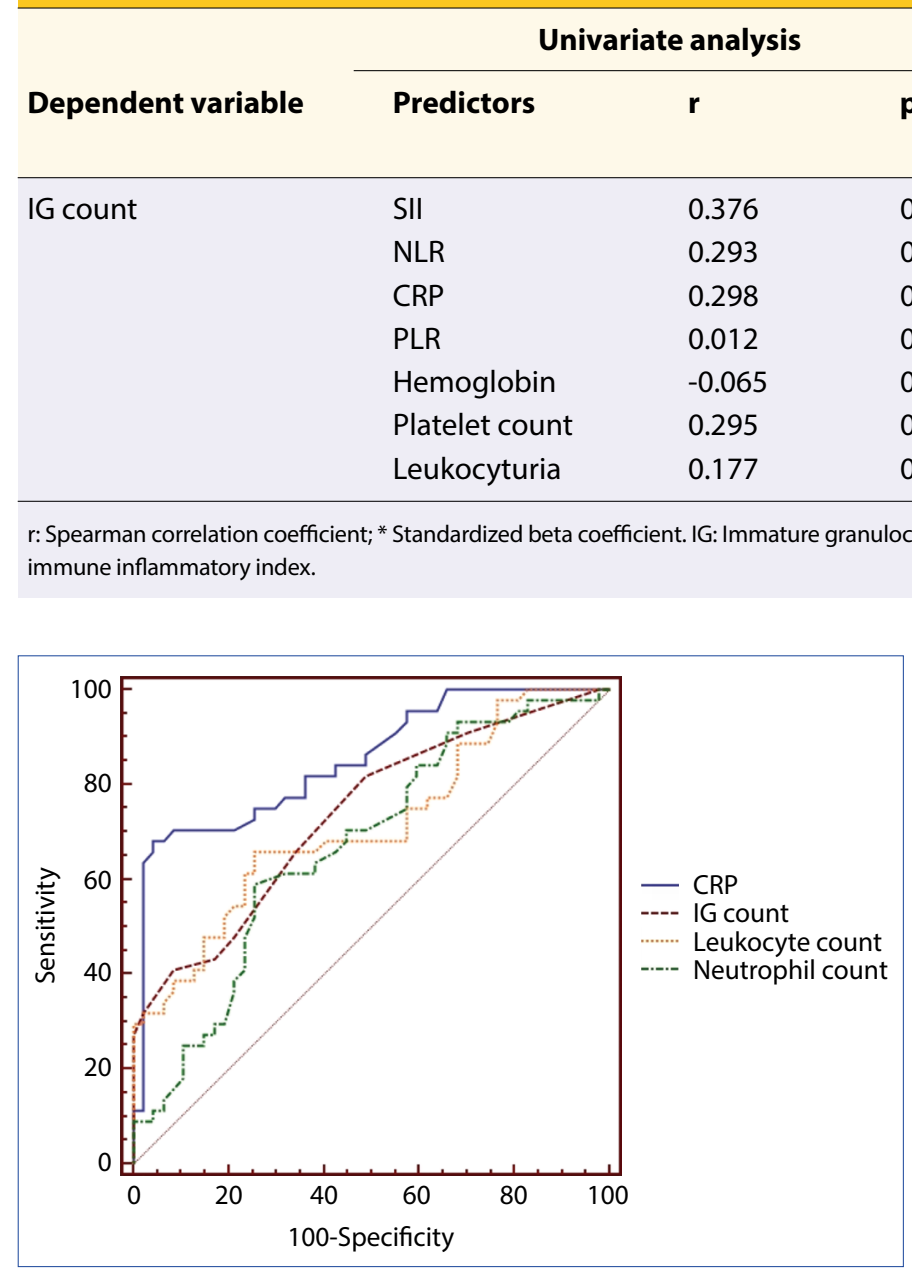

Figure 1. Comparison of the receiver operating characteristic $(R O C)$ curve analyses. The area under ROC curves for C-reactive protein (CRP), immature granulocyte (IG) count, and leukocyte and neutrophil counts were 0.851 (95\% confidence interval [Cl]: 0.761-0.917), 0.736 (95\% Cl: $0.633-0.823), 0.714$ (95\% Cl: $0.609-0.804)$, and 0.670 (95\% Cl: 0.564-0.765), respectively. The cut-off value for IG count as a predictor of urinary tract infection was $>0.03$ with a sensitivity of $65.45 \%(95 \%$ $\mathrm{Cl}: 51.4-77.8)$ and a specificity of $65.96 \%(95 \% \mathrm{Cl}: 50.7-79.1)$.

The IG count is a relatively new marker in the $C B C$ to potentially detect inflammation or bacterial infection. Henriot et al. [5] compared the IG count in children with viral or bacterial infections with those observed in healthy children. A significant difference was seen between children with viral $(p=0.002)$ or bacterial $(p<0.001)$ infection and the healthy group. Nierhaus et al. [10] evaluated 70 children with systemic inflammatory response syndrome (SIRS) and reported a significant difference in the IG count between the infected and noninfected patients $(p<0.001)$ with a sensitivity of $89.2 \%$ and a specificity of $76.4 \%$, notably in the first 48 hours. They also found that the IG count was a more indicative marker than other clinical parameters, including the levels of CRP level, lipopolysaccharide-binding protein, and interleukin 6 , with a low sensitivity value of $68 \%$.
There are very limited data on the relationship between IG and UTI. Yoon et al. [11] investigated the role of the delta neutrophil index (DNI), a parameter that illustrates the ratio of IG to total the neutrophil count, in infants with bacteremia associated with a febrile UTI. The authors found that the DNI had a moderate diagnostic value (AUC: 0.727 ), with an optimal cut-off value of $1.2 \%$ to detect bacteremia in infants with a UTI. Significantly higher DNI values were observed in infants with a UTI and concomitant bacteremia $(p=0.006)$ than those with a UTI and no bacteremia. Among infants and young children with a febrile UTI, the DNI was found to be significantly higher in the presence of vesicoureteral reflux (VUR) $(p<0.01)$ [12]. In this study, we found significantly higher IG counts in the patient group than in the control group $(p=0.001)$. In all, $40 \%$ of the patients had a fever and $25 \%$ had VUR. Due to the relatively small number of patients in this study, we could not conduct subgroup analysis of correlations between IG count and fever or VUR.

Inflammation is the first response of the immune system to an infection, and is associated with increased leukocyte and neutrophil counts [13]. The leukocyte and neutrophil counts of the patient group in this study were significantly higher than those of the control group ( $p=0.001)$. Though most UTIs are of bacterial origin, there was no significant difference in the lymphocyte count ( $p=0.155$ ).

Defective iron homeostasis and the suppressive effects of proinflammatory cytokines on erythropoiesis may cause anemia associated with inflammation. Recent epidemiological studies have demonstrated an association between anemia and infection, chronic illnesses, obesity, aging, kidney failure, cancer, and autoimmune diseases [14]. A significant relationship between anemia and UTI has been reported in previous studies. Certain inflammatory markers known to be elevated in patients with a febrile UTI, such as plasma neutrophil gelatinase-associated lipocalin and CRP, have been found to be negatively correlated with hemoglobin levels (both $p<0.001$ ). In addition, the presence of anemia has also been found to be an independent risk factor for renal scar- 
ring in children with a febrile UTI [15]. In the present study, the hemoglobin level was significantly lower in the patient group ( $\mathrm{p}=0.004)$.

Platelets play a crucial role in the inflammatory process by synthesizing various factors captured at the surface or in alpha granules, leading to a systemic immune response [16]. The MPV has been studied as an inflammatory marker related to UTI. Lee et al. [17] determined that the MPV was an acute phase reactant in children with a UTI. They found a significant difference in the MPV level between a group with acute pyelonephritis (APN) and a lower UTI group $(p=0.011)$, while the platelet count $(p=0.742)$ did not differ significantly between the groups. The MPV may increase with mild inflammation due to large platelets in the peripheral blood or decrease in the presence of severe inflammation as a result of the consumption of large platelets [18]. In this study, the platelet count was significantly higher in the patient group $(p=0.035)$ compared with that of the control group, while no significant difference was seen in the MPV $(p=0.370)$.

The assessment of the NLR, PLR, and SII as novel inflammatory markers to evaluate systemic inflammation in various diseases continues. Can et al. [19] investigated the role of the NLR and the PLR in 78 neonates with early-onset sepsis and reported a significant between-group difference in both values $(p=0.020$ and $p=0.001$, respectively). In another study, Loonen et al. [20] evaluated 125 adult patients with SIRS who presented at the emergency department. Of 125 patients, 27 with a positive blood culture had a significantly higher NLR value $(p<0.001)$ than the patients without positive blood culture. The prognostic value of the NLR has also been investigated as a means to predict APN in children with DMSA scan defects. The NLR was significantly higher in the group of those with APN and a DMSA defect $(p<0.001)$ and was determined to be an independent predictive factor for renal scarring (odds ratio: 1.603; 95\% Cl:1.263-2.035; $\mathrm{p}<0.001)$. Researchers have also reported similar predictive values for the NLR and CRP level (AUC: 0.713 and 0.726 , respectively) [21].

The SII, based on peripheral lymphocyte, neutrophil, and platelet counts, has been suggested as a better index of the local immune response and systemic inflammation because of its high prognostic value in various tumors $[22,23]$. To our knowledge, there is no study evaluating the role of SII in children with UTI. We found no significant difference between the NLR, PLR, or SIl values in our study groups.

However, univariate analysis did reveal a significant correlation between the IG count and the NLR $(r=0.293 ; p=0.030)$, SII $(r=0.376 ; p=0.005)$, platelet count $(r=0.295 ; p=0.029)$, and the CRP level $(r=0.298 ; p=0.040)$. No correlations were seen between the IG count and leukocyturia, nitrite, or leukocyte esterase values. Additional research with a larger group of patients would be useful in this area.

CRP is one of the inflammatory markers used most often to diagnose bacterial infection. Yet, it is noteworthy to recall that the CRP level can also increase in many other conditions, such as viral infection, non-infectious inflammatory diseases (e.g., autoimmune and rheumatic disorders), and myocardial infarction [24]. The predictive value of CRP in UTI has been investigated alone or in combination with other parameters in several studies [25-27]. We recorded the highest AUC values for the IG count and CRP level (Fig. 1). The sensitivity and specificity of the IG count to predict a UTI was $65.45 \%$ and $65.96 \%$, respectively, using a cut-off value of $>0.03$.

Lee et al. [12] reported a sensitivity value of $<50 \%$ and a moderate specificity ranging from $70 \%$ to $90 \%$ for the DNI as a predictor of UTI. In contrast, high sensitivity values of $82 \%$ to $95 \%$ have been reported in other studies examining the IG count and the DNI in patients with a UTI [10,28]. This rather significant sensitivity difference may be related to the adult age of the study populations or the presence of systemic infection, such as bacteremia or sepsis, in selected patients. Therefore, further studies are needed to evaluate the diagnostic accuracy of the IG count in a larger pediatric population with a UTI.

Our findings suggest that the IG count may be most valuable as an additional test, rather than an absolute parameter, in managing a UTI, given that the predictive value of the IG count was not superior to CRP in the diagnosis.

The primary strength of this study is that, to our knowledge, this is the first published research to evaluate the predictive value of the IG count in children with a UTI. The use of an ageand gender-homogenized control group is also valuable.

Nonetheless, the study does have some limitations: the number of patients was too small to perform subgroup analyses, e.g., febrile vs. afebrile or a comparison based on VUR. Sensitivity and specificity values for the IG count calculated with a larger number of patients would be more accurate. Secondly, this was a cross-sectional study. A determination of these values at different points in time would provide a better understanding of the role of the IG count in a UTI.

\section{Conclusion}

We found that the IG count was significantly elevated in children with a UTI. This value can be determined quickly and easily using a cost-effective and readily available method, and was independently predicted by CRP. The results of this research may prove useful to the algorithms used in the diagnosis of a UTI; however, further studies are needed to more completely evaluate the relationship between the IG count and other inflammatory parameters used in the diagnosis of a UTI.

Conflict of Interest: None declared.

Ethics Committee Approval: This study was approved by the Koc University Biomedical Research Ethics Committee. (Date: 24.04.202, No: 2020.151.IRB2.041)

Financial Disclosure: None declared.

Peer-review: Externally peer-reviewed. 
Authorship Contributions: Concept - S.I., K.E.P.; Design - S.I., M.T.; Supervision - S.I., M.T.; Funding - None; Materials - S.I., H.K.C.; Data collection \&/or processing - S.I., M.T., H.K.C.; Analysis and/or interpretation - A.B.; Literature search - H.K.C.; Writing S.I., M.T., I.B.; Critical review - S.I., K.E.P., I.B.

\section{References}

1. Catal F, Bavbek N, Bayrak O, Uz E, Isik B, Karabel M, et al. Platelet parameters in children with upper urinary tract infection: is there a specific response? Ren Fail 2008;30(4):377-81. [CrossRef]

2. Robinson JL, Finlay JC, Lang ME, Bortolussi R; Canadian Paediatric Society, Infectious Diseases and Immunization Committee, Community Paediatrics Committee. Urinary tract infections in infants and children: diagnosis and management. Paediatr Child Health 2014;19(6):315-25. [CrossRef]

3. Schroeder AR, Chang PW, Shen MW, Biondi EA, Greenhow TL. Diagnostic accuracy of the urinalysis for urinary tract infection in infants $<3$ months of age. Pediatrics 2015;135(6):965-71.

4. Aringer M. Inflammatory markers in systemic lupus erythematosus. J Autoimmun 2020;110:102374. [CrossRef]

5. Henriot I, Launay E, Boubaya M, Cremet L, Illiaquer M, Caillon $\mathrm{H}$, et al. New parameters on the hematology analyzer $\mathrm{XN}-10$ (SysmexTM) allow to distinguish childhood bacterial and viral infections. Int J Lab Hematol 2017;39(1):14-20. [CrossRef]

6. Ustundag Y, Huysal K, Karadag Gecgel S, Unal D. Relationship between $\mathrm{C}$-reactive protein, systemic immune-inflammation index, and routine hemogram-related inflammatory markers in low-grade inflammation. Int J Med Biochem 2018;1(1):24-8.

7. Ansari-Lari MA, Kickler TS, Borowitz MJ. Immature granulocyte measurement using the Sysmex XE-2100. Relationship to infection and sepsis. Am J Clin Pathol 2003;120(5):795-9. [CrossRef]

8. Briggs C, Harrison P, Grant D, Staves J, MacHin SJ. New quantitative parameters on a recently introduced automated blood cell counter--the XE 2100. Clin Lab Haematol 2000;22(6):34550. [CrossRef]

9. Incir S. The role of immature granulocytes and inflammatory hemogram indices in the inflammation. Int J Med Biochem. 2020;3(3):125-30. [CrossRef]

10. Nierhaus A, Klatte S, Linssen J, Eismann NM, Wichmann D, Hedke J, et al. Revisiting the white blood cell count: immature granulocytes count as a diagnostic marker to discriminate between SIRS and sepsis--a prospective, observational study. BMC Immunol 2013;14:8. [CrossRef]

11. Yoon SH, Shin H, Lee KH, Kim MK, Kim DS, Ahn JG, et al. Predictive factors for bacteremia in febrile infants with urinary tract infection. Sci Rep 2020;10(1):4469. [CrossRef]

12. Lee JW, Kim SH, Park SJ, Lee KH, Park JH, Kronbichler A, et al. The value of delta neutrophil index in young infants with febrile urinary tract infection. Sci Rep 2017;7:41265. [CrossRef]

13. Fensterl V, Sen GC. Interferons and viral infections. Biofactors 2009;35(1):14-20. [CrossRef]
14. Fraenkel PG. Anemia of inflammation: a review. Med Clin North Am 2017;101(2):285-96. [CrossRef]

15. Lee $\mathrm{JH}$, Yim HE, Yoo KH. Associations of plasma neutrophil gelatinase-associated lipocalin, anemia, and renal scarring in children with febrile urinary tract infections. J Korean Med Sci 2020;35(10):e65. [CrossRef]

16. Müller-Newen G, Stope MB, Kraus T, Ziegler P. Development of platelets during steady state and inflammation. J Leukoc Biol 2017;101(5):1109-17. [CrossRef]

17. Lee IR, Shin Jl, Park SJ, Oh JY, Kim JH. Mean platelet volume in young children with urinary tract infection. Sci Rep 2015;5:18072. [CrossRef]

18. Gasparyan AY, Sandoo A, Stavropoulos-Kalinoglou A, Kitas GD. Mean platelet volume in patients with rheumatoid arthritis: the effect of anti-TNF-a therapy. Rheumatol Int 2010;30(8):1125-9. [CrossRef]

19. Can E, Hamilcikan Ş, Can C. The value of neutrophil to lymphocyte ratio and platelet to lymphocyte ratio for detecting early-onset neonatal Sepsis. J Pediatr Hematol Oncol 2018;40(4):e229-32. [CrossRef]

20. Loonen AJ, de Jager CP, Tosserams J, Kusters R, Hilbink M, Wever PC, et al. Biomarkers and molecular analysis to improve bloodstream infection diagnostics in an emergency care unit. PLoS One 2014;9(1):e87315. [CrossRef]

21. Han SY, Lee IR, Park SJ, Kim JH, Shin JI. Usefulness of neutrophillymphocyte ratio in young children with febrile urinary tract infection. Korean J Pediatr 2016;59(3):139-44. [CrossRef]

22. Shao Y, Geng Y, Gu W, Ning Z, Huang J, Pei H, et al. Assessment of lymph node ratio to replace the pn categories system of classification of the tnm system in esophageal squamous cell carcinoma. J Thorac Oncol 2016;11(10):1774-84. [CrossRef]

23. Geng Y, Shao Y, Zhu D, Zheng X, Zhou Q, Zhou W, et al. Systemic immune-inflammation index predicts prognosis of patients with esophageal squamous cell carcinoma: a propensity score-matched analysis. Sci Rep 2016;6:39482. [CrossRef]

24. McMaster P, Park DY, Shann F, Cochrane A, Morris K, Gray J, et al. Procalcitonin versus $C$-reactive protein and immature-to-total neutrophil ratio as markers of infection after cardiopulmonary bypass in children. Pediatr Crit Care Med 2009;10(2):217-21.

25. Jung SJ, Lee JH. Prediction of cortical defect using c-reactive protein and urine sodium to potassium ratio in infants with febrile urinary tract infection. Yonsei Med J 2016;57(1):103-10.

26. Kuo HC, Liu HT, Tyagi $P$, Chancellor MB. Urinary nerve growth factor levels in urinary tract diseases with or without frequency urgency symptoms. Low Urin Tract Symptoms 2010;2(2):88-94.

27. Xu RY, Liu HW, Liu JL, Dong JH. Procalcitonin and C-reactive protein in urinary tract infection diagnosis. BMC Urol 2014;14:45. [CrossRef]

28. Park HJ, Ha YJ, Pyo JY, Park YB, Lee SK, Lee SW. Delta neutrophil index as an early marker for differential diagnosis of adult-onset Still's disease and sepsis. Yonsei Med J 2014;55(3):753-9. 